\title{
Association of self-reported sleep duration and quality with BaPWV levels in hypertensive patients
}

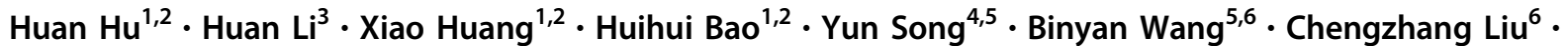

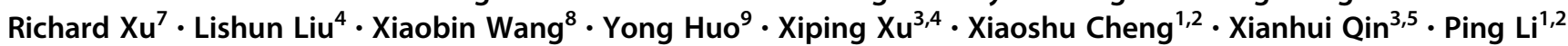

Received: 15 December 2019 / Revised: 2 May 2020 / Accepted: 3 June 2020 / Published online: 16 July 2020

(c) The Author(s) 2020. This article is published with open access

\begin{abstract}
The association between sleep conditions and arterial stiffness remains inconclusive. We aimed to investigate the relationship of sleep duration and quality with brachial-ankle pulse-wave velocity (baPWV) in hypertensive patients. A total of 14,485 hypertensive adults were included in this cross-sectional analysis. Information about sleep duration and quality was obtained via questionnaire. A baPWV level $\geq 1800 \mathrm{~cm} / \mathrm{s}$ was defined as indicative of arterial stiffness. Compared with participants with a sleep duration $<8 \mathrm{~h}$ per day, participants with a sleep duration $\geq 8 \mathrm{~h}$ per day had a significantly higher baPWV level $(\beta=13.7 \mathrm{~cm} / \mathrm{s} ; 95 \%$ CI: 3.9, 23.5) and a nonsignificantly higher prevalence of arterial stiffness $(39.7 \%$ vs. $33.0 \%$; OR, 1.08; 95\% CI: 0.99-1.19). Similarly, compared with participants with good or medium sleep quality, participants with poor sleep quality had a significantly higher baPWV level $(\beta=16.3 \mathrm{~cm} / \mathrm{s} ; 95 \%$ CI: $0.1,32.6)$ and a nonsignificantly greater prevalence of arterial stiffness $(36.6 \%$ vs. $35.3 \%$; OR, 1.13 ; 95\% CI: 0.97-1.32). When sleep duration and quality were examined jointly, participants with a sleep duration $\geq 8 \mathrm{~h}$ and/or poor sleep quality had a significantly higher baPWV level $(\beta=14.4 \mathrm{~cm} / \mathrm{s} ; 95 \%$ CI: $5.3,23.4)$ and a greater prevalence of arterial stiffness $(38.8 \%$ vs. 32.7\%; OR, 1.10; 95\% CI: 1.01-1.20) than those with a sleep duration $<8 \mathrm{~h}$ and good/medium sleep quality. In summary, among hypertensive patients, a longer sleep duration ( $\geq 8 \mathrm{~h}$ per day) and poor sleep quality were associated with higher baPWV levels and a higher prevalence of arterial stiffness.
\end{abstract}

Keywords Hypertension $\cdot$ Sleep duration $\cdot$ Sleep quality $\cdot$ Arterial stiffness $\cdot$ Brachial-ankle pulse-wave velocity

\section{Introduction}

Supplementary information The online version of this article (https:// doi.org/10.1038/s41440-020-0509-y) contains supplementary material, which is available to authorized users.

\section{Xianhui Qin}

pharmaqin@126.com

$\triangle$ Ping Li

lipingsydney@163.com

1 Department of Cardiology, the Second Affiliated Hospital of Nanchang University, Nanchang, China

2 Center for Prevention and Treatment of Cardiovascular Diseases, the Second Affiliated Hospital of Nanchang University, Nanchang, China

3 National Clinical Research Study Center for Kidney Disease; State Key Laboratory for Organ Failure Research; Renal Division, Nanfang Hospital, Southern Medical University, Guangzhou, China
Arterial stiffness is a subclinical measure of vascular disease, and increased arterial stiffness indicates the deterioration of arterial blood vessel elasticity. To date, arterial stiffness has been reported to be an independent predictor of

4 Beijing Advanced Innovation Center for Food Nutrition and Human Health, College of Food Science and Nutritional Engineering, China Agricultural University, Beijing, China

5 Institute of Biomedicine, Anhui Medical University, Hefei, China

6 Shenzhen Evergreen Medical Institute, Shenzhen, China

7 Krieger School of Arts and Sciences, Johns Hopkins University, Baltimore, USA

8 Department of Population, Family and Reproductive Health, Johns Hopkins University Bloomberg School of Public Health, Baltimore, USA

9 Department of Cardiology, Peking University First Hospital, Beijing, China 
both atherosclerosis and cardiovascular diseases (CVDs) $[1,2]$. Thus, a better understanding of the potential risk factors for increased arterial stiffness may help to prevent the onset of arterial stiffness and related CVDs.

Generally, increased arterial stiffness results from the deterioration of vascular wall structure and function. Previous studies have suggested that chronic sleep fragmentation and sleep restriction may be involved in the progression of endothelial dysfunction and structural vascular changes [3, 4]. Thus, there may be a link between sleep disturbances and arterial stiffness. In fact, several previous studies have investigated the association between abnormal sleep conditions and arterial stiffness, but they have reported inconsistent findings [5-9]. Therefore, the relationship between sleep conditions and arterial stiffness remains inconclusive.

Hypertension is an important risk factor for CVDs and chronic kidney diseases [10, 11]. Hypertension is usually accompanied by the deterioration of sleep quality and increased arterial stiffness $[12,13]$. To date, few studies have been conducted to explore the association between sleep disturbances and arterial stiffness among the general hypertensive population. Brachial-ankle pulse-wave velocity (baPWV), a convenient measurement to assess the stiffness of combined large arteries and peripheral arteries, is widely used as a simple noninvasive measure of arterial stiffness to screen subclinical vascular damage in large populations [14, 15]. A higher baPWV value represents a higher level of arterial stiffness, and an increasing number of studies have been performed using the baPWV level to assess arterial stiffness. Therefore, to fill the above knowledge gap, our current study aimed to investigate the relationship between sleep duration and quality with baPWV levels in hypertensive patients using data from the China Stroke Primary Prevention Trial (CSPPT) at site exit visits.

\section{Methods}

\section{Study participants and design}

All participants of this study were from the CSPPT (URL: https://www.clinicaltrials.gov; Unique identifier: NCT007 94885). The methods and major results of the CSPPT [16-19] have been reported previously. Briefly, the CSPPT was a multicenter, randomized, double-blind, controlled trial performed from May 2008 to August 2013 in 32 communities in China. The inclusion criteria were participants aged 45 to 75 years old who had hypertension, defined as seated resting systolic blood pressure (SBP) of $140 \mathrm{mmHg}$ or higher or diastolic blood pressure of $90 \mathrm{mmHg}$ or higher at both the screening and recruitment visits or the use of antihypertensive medication. The major exclusion criteria included a history of physician-diagnosed stroke, myocardial infarction, heart failure, coronary revascularization, or congenital heart disease. The CSPPT showed that a combination of enalapril and folic acid is more effective in reducing the risk of first stroke than enalapril alone among a total of 20,702 Chinese hypertensive patients without major CVDs after a median of 4.5 years of follow-up.

Our current study was a cross-sectional analysis using data from the CSPPT at the site exit visit. Overall, of the 20,702 enrolled participants in the CSPPT, 15,197 participants completed the exit site visit and had baPWV measurements. Of those, 14,485 participants with detailed information about sleep duration and quality and with an ABI measurement $\geq 0.9$ were included in the current study (Supplemental fig. 1). This study was approved by the Ethics Committee of the Institute of Biomedicine, Anhui Medical University, Hefei, China. All participants provided written informed consent.

\section{Measurement of baPWV}

The baPWV of each participant was measured using an automatic noninvasive vascular function analyzer (form PWV/ABI, BP-203RPE; Omron-Colin, Japan) [20, 21]. Briefly, after $\geq 15 \mathrm{~min}$ of rest in the supine position, the bilateral brachial arteries and tibial arteries of each subject were wrapped with four oscillometric cuffs connected to a plethysmographic sensor. A phonocardiogram, electrocardiogram, and pulse-wave as well as the blood pressure at both ankles and the brachia can be recorded with the analyzer. Blood pressure, heart rate (HR), and ABI were measured concurrently with PWV. ABI was reported as the ratio of the ankle SBP to the brachial SBP. The value of baPWV was the propagation distance of the pulse wave divided by the pulse-wave propagation time.

According to previous studies [22, 23], a baPWV $\geq 1800 \mathrm{~cm} / \mathrm{s}$ was defined as arterial stiffness in this study.

\section{Data collection}

Each participant was interviewed using a standardized questionnaire designed specifically for the present study by trained research staff. The question about sleep duration was phrased as follows: "How long do you usually sleep every day?", and the following three responses choices were given: $<5,5-8$ and $\geq 8 \mathrm{~h}$. The question about sleep quality was phrased as "How do you describe your sleep quality?", and 3 response choices (poor, medium, and good) were provided. The question about smoking status was phrased as "How do you describe your smoking?", and 3 response choices (never, ever, and current) were provided. The 
question about alcohol consumption status was phrased as "How do you describe your drinking?", and 3 response choices (never, ever, and current) were provided. The question about physical activity was phrased as "How do you describe your daily physical activity level?", and 3 response choices (low, moderate, and high) were provided [24-26].

Seated blood pressure was measured using a mercury manometer using the standard method and appropriately sized cuffs by trained research staff after the subjects had been seated for $10 \mathrm{~min}$. Triplicate measurements on the same arm were taken, with at least 2 min between readings. Each patient's SBP and DBP were calculated as the mean of the three independent measurements.

\section{Laboratory assays}

Serum fasting glucose, lipids, total homocysteine (tHcy), and creatinine levels were measured using automated clinical analyzers (Beckman Coulter, USA) at the core laboratory of the National Clinical Research Center for Kidney Disease, Guangzhou, China.

\section{Statistical analysis}

Baseline characteristics are presented as the means \pm standard deviations (SDs) for continuous variables and proportions for categorical variables according to sleep duration or sleep quality. The differences in population characteristics were compared using ANOVA tests, signed rank tests, or chi-square tests.

The relationships of sleep duration and/or sleep quality with baPWV levels and arterial stiffness (baPWV $\geq$ $1800 \mathrm{~cm} / \mathrm{s}$ ) were evaluated using linear regression models and logistic regression models, respectively, without and with adjustments for age, sex, body mass index (BMI), triglycerides (TGs), total cholesterol (TC), high-density lipoprotein cholesterol (HDL-C), fasting glucose, tHcy, creatinine, SBP, HR, study treatment group, study centers, smoking status, alcohol consumption, and physical activity. Moreover, any potential factors modifying the relationship were explored by subgroup analyses via the assessments of the following variables, including sex, age $(<65$ vs. $\geq 65$ years), BMI ( $<24$ vs. $\geq 24 \mathrm{~kg} / \mathrm{m}^{2}$ ), SBP ( $<140$ vs. $\geq 140 \mathrm{mmHg}$ ), current smoking (yes vs. no), current alcohol consumption (yes vs. no), physical activity (low, moderate, and high), TC (<5.2 vs. $\geq 5.2 \mathrm{mmol} / \mathrm{L})$, fasting glucose ( $<6.1$ vs. $\geq 6.1 \mathrm{mmol} / \mathrm{L}$ or diabetes $)$, and tHcy levels ( $<12.2$ [median] vs. $\geq 12.2 \mu \mathrm{mol} / \mathrm{L})$.

A two-tailed $P<0.05$ was considered to be statistically significant in all analyses. $\mathrm{R}$ software (version 3.4.3, http://www.R-project.org) was used for all statistical analyses.

\section{Results}

\section{Study participants and baseline characteristics}

Overall, the mean age of all participants was 64.4 (SD: 7.4) years, $40.2 \%$ of them were men, and the mean baPWV was $1727.4 \mathrm{~cm} / \mathrm{s}$ (SD, 340.3). The general characteristics of the study participants are presented by sleep duration categories $(<5,5-8$ and $\geq 8 \mathrm{~h})$ in Table 1 . Participants with a longer sleep duration tended to be older; were more likely to be male, smokers and alcohol consumers; had higher total homocysteine, creatinine, BP levels, and heart rate levels; and had lower sleep quality and HDL-C levels at baseline.

Moreover, participants with poor sleep quality tended to be younger; were more likely to be females; had a higher heart rate, HDL-C, and TC levels; and had shorter sleep duration, lower BMI, total homocysteine, and creatinine levels at baseline (Table 2).

\section{Relationship of sleep duration with baPWV and arterial stiffness}

In the multivariate models, compared with participants with a sleep duration of 5-8 h, a significantly higher baPWV level $(\beta, 14.0 \mathrm{~cm} / \mathrm{s} ; 95 \% \mathrm{CI}: 4.1,23.8)$ and a nonsignificantly greater prevalence of arterial stiffness (OR, 1.09; 95\% CI: 0.99-1.19) were found in those with a sleep duration $\geq 8 \mathrm{~h}$ (Table 3, Supplemental Table 1).

Due to the similar baPWV levels and arterial stiffness prevalence in participants with sleep durations of $<5$ and 5-8 h (Table 3, Supplemental Table 1), we combined these two groups into one group called sleep duration $<8 \mathrm{~h}$. Compared to participants with a sleep duration $<8 \mathrm{~h}$, a higher baPWV level $(\beta, 13.7 \mathrm{~cm} / \mathrm{s} ; 95 \% \mathrm{CI}: 3.9,23.5)$ and a greater prevalence of arterial stiffness (OR, 1.08; 95\% CI: 0.99-1.19) were found in those with a sleep duration $\geq 8 \mathrm{~h}$ (Table 3, Supplemental Table 1).

\section{Relationship of sleep quality with baPWV and arterial stiffness}

In the multivariate models, compared to participants with good quality, a higher baPWV level $(\beta, 15.5 \mathrm{~cm} / \mathrm{s} ; 95 \% \mathrm{CI}$ : $-1.7,37.8)$ and a greater prevalence of arterial stiffness (OR, 1.13; 95\% CI: 0.96-1.33) were found among those with poor sleep quality (Table 3, Supplemental Table 1).

Due to the similar baPWV levels and arterial stiffness prevalence in participants with good and medium sleep quality (Table 3, Supplemental Table 1), we combined these two groups into one group called the good/medium sleep quality group. Compared with participants with good/ medium sleep quality, a higher baPWV level $(\beta, 16.3 \mathrm{~cm} / \mathrm{s}$; 
Table 1 Characteristics of participants by sleep duration $(n=14,485)^{\mathrm{a}}$

\begin{tabular}{|c|c|c|c|c|}
\hline \multirow[t]{2}{*}{ Characteristics } & \multicolumn{3}{|c|}{ Sleep duration per day } & \multirow{2}{*}{$P$ value } \\
\hline & $<5 \mathrm{~h}$ & $5-8 \mathrm{~h}$ & $\geq 8 \mathrm{~h}$ & \\
\hline Number & 621 & 8667 & 5197 & \\
\hline Age (years) & $64.8 \pm 7.4$ & $63.6 \pm 7.3$ & $65.6 \pm 7.4$ & $<0.001$ \\
\hline Male, No. (\%) & $187(30.1)$ & $3402(39.3)$ & $2240(43.1)$ & $<0.001$ \\
\hline Body mass index $\left(\mathrm{kg} / \mathrm{m}^{2}\right)$ & $24.6 \pm 4.0$ & $25.0 \pm 3.7$ & $24.9 \pm 3.9$ & 0.020 \\
\hline Enalapril-folic acid group, $n(\%)$ & $304(49.0)$ & $4320(49.8)$ & $2590(49.8)$ & 0.910 \\
\hline Current smoker, $n(\%)$ & $95(15.3)$ & $1838(21.3)$ & $1116(21.5)$ & 0.002 \\
\hline Current alcohol drinker, $n(\%)$ & $97(16.2)$ & $1913(22.8)$ & $1195(24.0)$ & $<0.001$ \\
\hline Systolic blood pressure (mmHg) & $133.9 \pm 17.7$ & $135.2 \pm 17.2$ & $135.8 \pm 17.6$ & 0.022 \\
\hline Diastolic blood pressure $(\mathrm{mmHg})$ & $81.3 \pm 10.8$ & $82.2 \pm 10.9$ & $81.4 \pm 10.8$ & $<0.001$ \\
\hline Heart rate (bpm) & $76.8 \pm 11.5$ & $76.6 \pm 11.1$ & $77.2 \pm 11.7$ & 0.023 \\
\hline baPWV (cm/s) & $1733.1 \pm 358.1$ & $1704.9 \pm 330.4$ & $1764.3 \pm 351.1$ & $<0.001$ \\
\hline Physical activity, $n(\%)$ & & & & $<0.001$ \\
\hline Low & $307(49.4)$ & $3676(42.4)$ & $2433(46.9)$ & \\
\hline Moderate & $210(33.8)$ & $3707(42.8)$ & $2130(41.0)$ & \\
\hline High & $104(16.7)$ & $1277(14.7)$ & $630(12.1)$ & \\
\hline Sleep quality, $n(\%)$ & & & & $<0.001$ \\
\hline Good & $56(9.0)$ & $3076(35.5)$ & $3174(61.1)$ & \\
\hline Medium & $130(20.9)$ & $4747(54.8)$ & $1806(34.8)$ & \\
\hline Poor & $435(70.0)$ & $844(9.7)$ & $217(4.2)$ & \\
\hline \multicolumn{5}{|l|}{ Laboratory results } \\
\hline Creatinine $(\mu \mathrm{mol} / \mathrm{L})$ & $67.9 \pm 23.9$ & $67.9 \pm 23.7$ & $70.1 \pm 31.6$ & $<0.001$ \\
\hline Glucose $(\mathrm{mmol} / \mathrm{L})$ & $6.3 \pm 2.3$ & $6.2 \pm 1.9$ & $6.3 \pm 2.1$ & 0.001 \\
\hline Total cholesterol $(\mathrm{mmol} / \mathrm{L})$ & $5.4 \pm 1.2$ & $5.3 \pm 1.1$ & $5.3 \pm 1.1$ & $<0.001$ \\
\hline Triglyceride (mmol/L) & $1.7 \pm 1.5$ & $1.8 \pm 1.5$ & $1.8 \pm 1.4$ & 0.654 \\
\hline HDL-C (mmol/L) & $1.33 \pm 0.33$ & $1.28 \pm 0.31$ & $1.27 \pm 0.32$ & $<0.001$ \\
\hline Total homocysteine $(\mu \mathrm{mol} / \mathrm{L})$ & $13.2 \pm 5.0$ & $13.2 \pm 6.5$ & $14.0 \pm 8.3$ & $<0.001$ \\
\hline
\end{tabular}

$b a P W V$ brachial-ankle pulse-wave velocity, $H D L-C$ high-density lipoprotein cholesterol

${ }^{\mathrm{a}}$ Variables are presented as mean $\pm \mathrm{SD}$ or $n(\%)$
95\% CI: 0.1, 32.6) and a greater prevalence of arterial stiffness (OR, 1.13; 95\% CI: 0.97-1.32) were found in those with poor sleep quality (Table 3, Supplemental Table 1).

\section{Relationship of combined sleep duration and sleep quality with baPWV and arterial stiffness}

Compared with participants with a sleep duration of $<8 \mathrm{~h}$ and good/medium sleep quality, a higher baPWV level was found in those with sleep duration $<8 \mathrm{~h}$ and poor sleep quality $(\beta, 14.5 \mathrm{~cm} / \mathrm{s} ; 95 \% \mathrm{CI}:-1.7,30.8)$, sleep duration $\geq 8 \mathrm{~h}$ and good/medium sleep quality $(\beta, 12.8 \mathrm{~cm} / \mathrm{s} ; 95 \% \mathrm{CI}$ : $3.0,22.6$ ), or sleep duration $\geq 8 \mathrm{~h}$ and poor sleep quality $(\beta, 49.5 \mathrm{~cm} / \mathrm{s} ; 95 \%$ CI: 12.7, 86.3) (Table 4). Accordingly, compared with participants with a sleep duration $<8 \mathrm{~h}$ and good/medium sleep quality, those with a sleep duration $\geq 8 \mathrm{~h}$ and/or poor sleep quality had a significantly higher baPWV level $(\beta, 14.4 \mathrm{~cm} / \mathrm{s} ; 95 \%$ CI: 5.3, 23.4) (Table 4). Similar results were found for arterial stiffness (Supplemental Table 2).

\section{Stratified analyses by potential effect modifiers}

Stratified analyses were performed to further assess the association between combined sleep duration and sleep quality (sleep duration $\geq 8 \mathrm{~h}$ and/or poor sleep quality vs. sleep duration $<8 \mathrm{~h}$ and good/medium sleep quality) with baPWV and arterial stiffness in various subgroups (Table 5 and Supplemental Table 3). No significant interactions were found in the association in any of the subgroups, including sex, age ( $<65$ vs. $\geq 65$ years), BMI ( $<24$ vs. $\geq 24 \mathrm{~kg} / \mathrm{m}^{2}$ ), SBP (<140 vs. $\geq 140 \mathrm{mmHg}$ ), current alcohol consumption (no vs. yes), intensity of physical activity (low, moderate, and high), TC ( $<5.2$ vs. $\geq 5.2 \mathrm{mmol} / \mathrm{L})$, fasting glucose $(<6.1$ vs. $\geq 6.1 \mathrm{mmol} / \mathrm{L}$ or diabetes $)$ and tHcy $(<12.2$ [median] vs. $\geq 12.2 \mu \mathrm{mol} / \mathrm{L}$ ) subgroups (all $P$ for interactions $>0.05$ ). 
Table 2 Characteristics of participants by sleeping quality $(n=14,485)^{\mathrm{a}}$

\begin{tabular}{|c|c|c|c|c|}
\hline \multirow[t]{2}{*}{ Characteristics } & \multicolumn{3}{|l|}{ Sleep quality } & \multirow[t]{2}{*}{$P$ value } \\
\hline & Good & Medium & Poor & \\
\hline Number & 6306 & 6683 & 1496 & \\
\hline Age (years) & $64.6 \pm 7.4$ & $64.2 \pm 7.3$ & $63.9 \pm 7.4$ & $<0.001$ \\
\hline Male, No. (\%) & $2993(47.5)$ & $2448(36.6)$ & $388(25.9)$ & $<0.001$ \\
\hline Body mass index $\left(\mathrm{kg} / \mathrm{m}^{2}\right)$ & $25.0 \pm 3.8$ & $24.9 \pm 3.8$ & $24.7 \pm 3.8$ & 0.014 \\
\hline Enalapril-folic acid group, $n(\%)$ & $3146(49.9)$ & $3325(49.8)$ & $743(49.7)$ & 0.982 \\
\hline Current smoker, $n(\%)$ & $1496(23.8)$ & $1333(20.0)$ & $220(14.7)$ & $<0.001$ \\
\hline Current alcohol drinker, $n(\%)$ & 1594 (26.6) & $1383(21.3)$ & $228(15.6)$ & $<0.001$ \\
\hline Systolic blood pressure $(\mathrm{mmHg})$ & $135.4 \pm 17.5$ & $135.4 \pm 17.2$ & $135.0 \pm 17.3$ & 0.677 \\
\hline Diastolic blood pressure (mmHg) & $81.8 \pm 10.9$ & $81.9 \pm 10.8$ & $82.1 \pm 11.0$ & 0.713 \\
\hline Heart rate $(\mathrm{bpm})$ & $76.5 \pm 11.4$ & $77.0 \pm 11.3$ & $77.6 \pm 11.5$ & $<0.001$ \\
\hline baPWV $(\mathrm{cm} / \mathrm{s})$ & $1728.2 \pm 342.7$ & $1724.7 \pm 337.3$ & $1736.7 \pm 343.7$ & 0.453 \\
\hline Physical activity, $n(\%)$ & & & & $<0.001$ \\
\hline Low & $2961(47.0)$ & $2749(41.2)$ & $706(47.2)$ & \\
\hline Moderate & $2445(38.8)$ & $3067(45.9)$ & $535(35.8)$ & \\
\hline High & $895(14.2)$ & $862(12.9)$ & $254(17.0)$ & \\
\hline Sleep duration, $n(\%)$ & & & & $<0.001$ \\
\hline$<5 \mathrm{~h}$ & $56(0.9)$ & $130(1.9)$ & $435(29.1)$ & \\
\hline $5-8 \mathrm{~h}$ & $3076(48.8)$ & $4747(71.0)$ & $844(56.4)$ & \\
\hline$\geq 8 \mathrm{~h}$ & $3174(50.3)$ & $1806(27.0)$ & $217(14.5)$ & \\
\hline \multicolumn{5}{|l|}{ Laboratory results } \\
\hline Creatinine $(\mu \mathrm{mol} / \mathrm{L})$ & $70.5 \pm 31.7$ & $67.6 \pm 23.1$ & $66.1 \pm 18.1$ & $<0.001$ \\
\hline Glucose (mmol/L) & $6.3 \pm 2.0$ & $6.2 \pm 2.0$ & $6.3 \pm 2.0$ & 0.595 \\
\hline Total cholesterol (mmol/L) & $5.3 \pm 1.1$ & $5.3 \pm 1.1$ & $5.5 \pm 1.1$ & $<0.001$ \\
\hline Triglyceride $(\mathrm{mmol} / \mathrm{L})$ & $1.8 \pm 1.4$ & $1.8 \pm 1.5$ & $1.8 \pm 1.3$ & 0.896 \\
\hline HDL-C (mmol/L) & $1.27 \pm 0.31$ & $1.28 \pm 0.31$ & $1.31 \pm 0.32$ & $<0.001$ \\
\hline Total homocysteine $(\mu \mathrm{mol} / \mathrm{L})$ & $13.9 \pm 7.9$ & $13.3 \pm 6.7$ & $12.9 \pm 5.6$ & $<0.001$ \\
\hline
\end{tabular}

$b a P W V$ brachial-ankle pulse-wave velocity, $H D L-C$ high-density lipoprotein cholesterol

${ }^{\mathrm{a}}$ Variables are presented as mean $\pm \mathrm{SD}$ or $n(\%)$
However, a stronger association of combined sleep duration and sleep quality (sleep duration $\geq 8 \mathrm{~h}$ and/or poor sleep quality $v s$. sleep duration $<8 \mathrm{~h}$ and good/medium sleep quality) with baPWV was found in participants with a current smoking habit ( $\beta: 34.2 \mathrm{~cm} / \mathrm{s} ; 95 \%$ CI: 13.7, 54.6) than in those without a current smoking habit $(\beta: 9.3 \mathrm{~cm} / \mathrm{s}$; 95\% CI: $-0.8,19.4 ; P$-interaction $=0.049$ ).

\section{Discussion}

Our current study revealed that a longer sleep duration $(\geq 8 \mathrm{~h})$ or poor sleep quality was associated with higher baPWV levels and a greater prevalence of arterial stiffness in the general Chinese hypertensive population. Furthermore, current smoking was a modifier of this association.

To date, the relationship between sleep conditions and arterial stiffness remains inconclusive. Kim et al. [9] performed a large cross-sectional study among an apparently healthy population and found that extreme sleep durations $(<7$ or $\geq 7 \mathrm{~h}$ ) and poor subjective sleep quality were associated with an increased prevalence of higher baPWV. Cao et al. [27], using sleep duration $\geq 8 \mathrm{~h}$ as the reference, reported that there was no significant association between a sleep duration of $\leq 6$ or $6-8 \mathrm{~h}$ and arterial stiffness. However, participants with poor sleep quality and a sleep duration $\leq 6 \mathrm{~h}$ were more likely to have arterial stiffness than those with good quality sleep who slept for $\geq 8 \mathrm{~h}$ (OR, 2.59; 95\% CI: 1.58-4.24). Yoshioka et al. [5] revealed that a daily sleep duration $\geq 9 \mathrm{~h}$ was associated with elevated values of baPWV only among males in a large-scale Japanese study. Another cross-sectional study performed in Taiwan showed that long $(>8 \mathrm{~h})$, but not short $(<6 \mathrm{~h})$, sleep duration was associated with a higher risk of increased arterial stiffness in males [6]. The discrepancies among these findings may be due to the difference in study populations or the definitions of sleep duration or quality. Our study provided an opportunity to assess the relationship between sleep duration and 


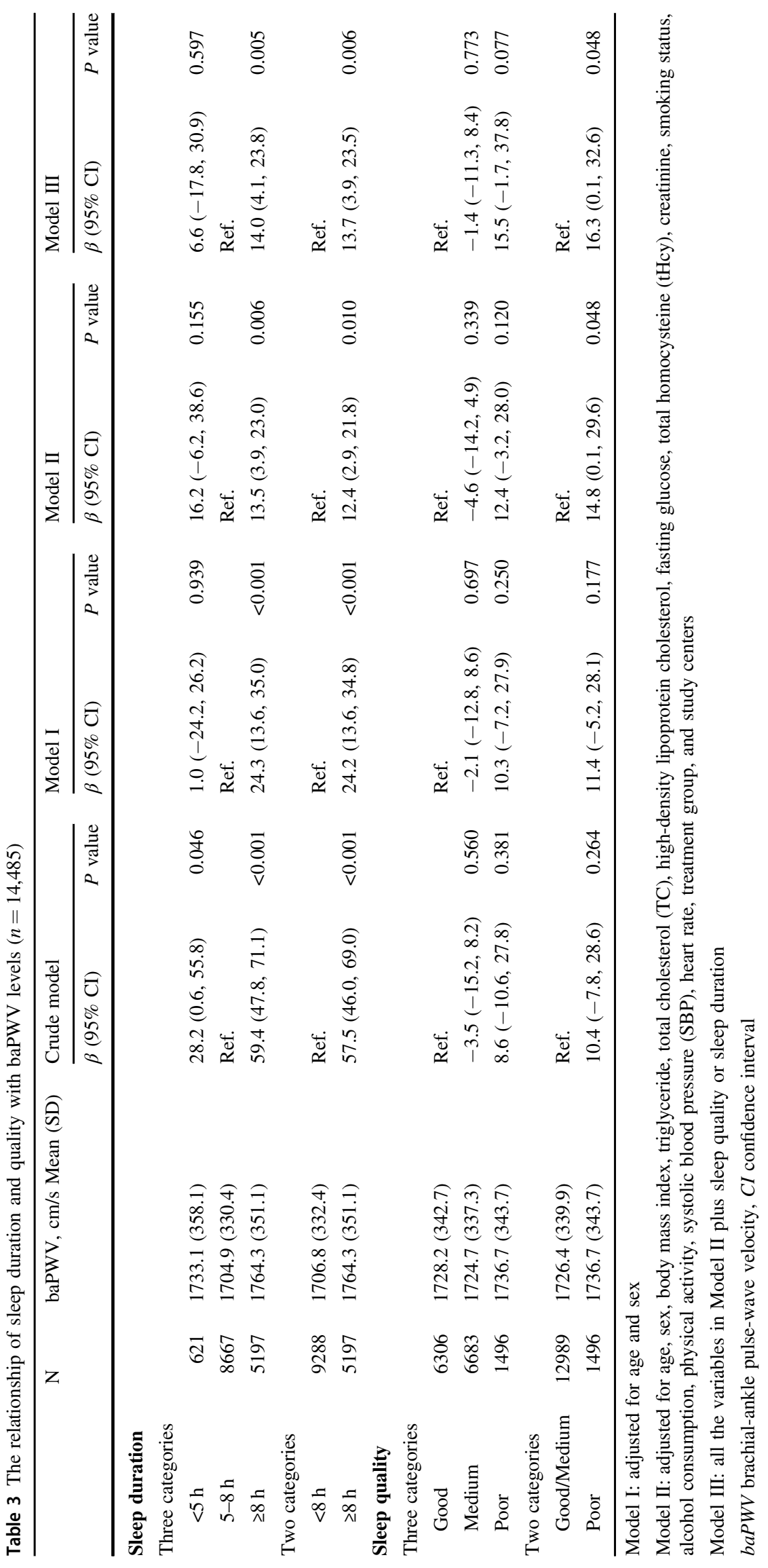




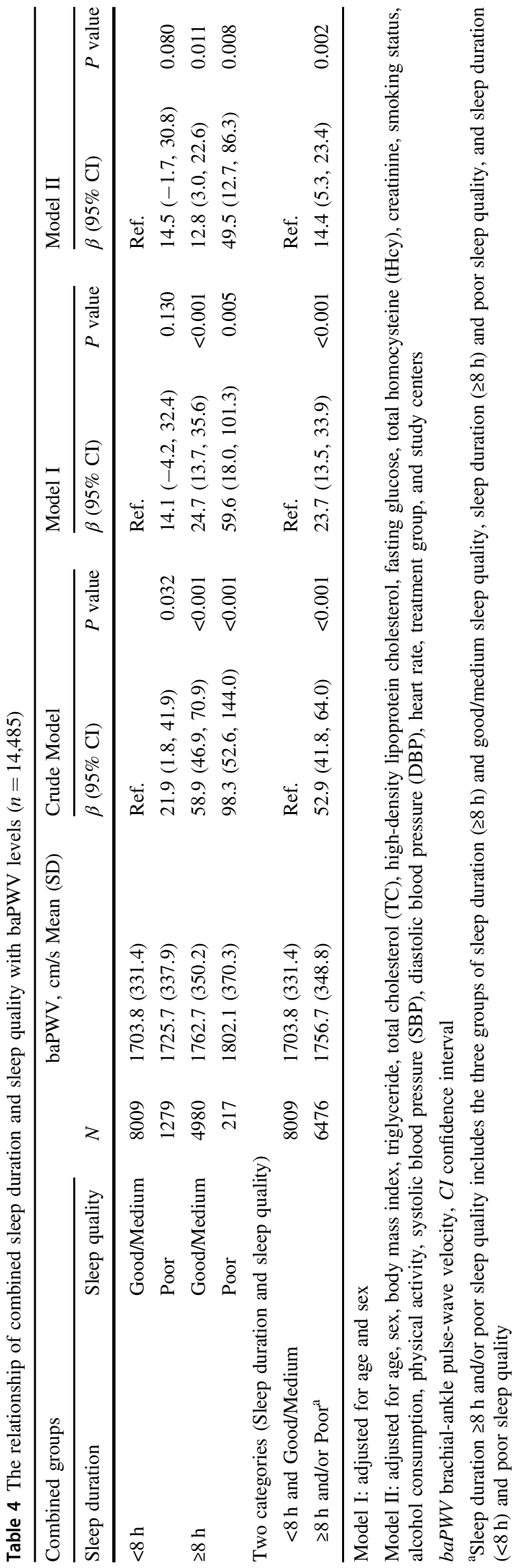

sleep quality with arterial stiffness in general hypertensionmanaged patients, with comprehensive adjustments for a series of important confounders.

With a large sample size of 14,485 hypertensive adults, the present study explored the separate effects of sleep duration and sleep quality on baPWV levels with further adjustment for sleep quality or sleep duration, providing several novel insights into the field of the investigation of sleep and arterial stiffness. First, a sleep duration $\geq 8 \mathrm{~h}$ was positively associated with elevated baPWV levels and increased arterial stiffness in hypertensive patients. Consistently, a recent meta-analysis by Jike et al. [28] including $5,134,036$ subjects from 137 prospective cohort studies concluded that a self-reported longer sleep duration was significantly associated with mortality (RR, 1.39; 95\% CI, $1.31,1.47)$, CVDs $(1.25 ; 1.14,1.37)$, stroke $(1.46 ; 1.26$, $1.69)$ and coronary heart disease $(1.24 ; 1.13,1.37)$. However, the exact mechanisms behind the relationship of long sleep duration and these health outcomes have not yet been elucidated. Grandner et al. [29] indicated that sleep fragmentation, fatigue, immune functionality, lack of challenges, depression, or underlying disease processes, such as sleep apnea, may be potential mechanisms of the association between long sleep and mortality. The present study found a positive relationship between self-reported longer sleep duration ( $\geq 8 \mathrm{~h}$ ) and higher baPWV levels, providing a potential mechanism linking long sleep duration and poor health outcomes via mediation of arterial stiffness. Irwin et al. [30] indicated that longer sleep durations $(>8 \mathrm{~h}$ per night), but not shorter sleep durations ( $<7 \mathrm{~h}$ per night), were associated with elevated levels of systemic inflammation markers, which could increase arterial stiffness by enabling vascular fibrosis and smooth muscle cell proliferation [31]. Therefore, the inflammatory pathway may be one of the mechanisms involved in the association between longer sleep duration and arterial stiffness. Our current study failed to find a similar association between short sleep duration and the risk of arterial stiffness, which might be partly due to the difference in study populations and the definition of sleep duration. More studies concerning the association between objectively measured sleep duration and baPWV levels are needed to confirm our findings.

Second, the present study also found a positive association between poor sleep quality and increased baPWV levels in hypertensive patients. Consistently, the study by Osonoi et al. [7], including 724 patients with type 2 diabetes mellitus, revealed that poor sleep quality, assessed by the Pittsburg Sleep Quality Index (PSQI), was associated with increased arterial stiffness. Moreover, the study by Guney et al. [32], including 199 patients with chronic kidney disease, found that participants with poor sleep quality, assessed by the PSQI, had higher PWV levels than those with good sleep quality. However, only 
Table 5 Stratified analysis of the association between combined sleep duration and quality (sleep duration $\geq 8 \mathrm{~h}$ and/or poor sleep quality $v s$. sleep duration $<8 \mathrm{~h}$ and good/medium sleep quality) with baPWV levels

\begin{tabular}{|c|c|c|c|c|c|}
\hline \multirow[t]{2}{*}{ Subgroups } & \multirow[t]{2}{*}{$N$} & \multirow{2}{*}{$\begin{array}{l}<8 \mathrm{~h} \text { and good/ } \\
\text { medium } \\
\text { baPWV, cm/s } \\
\text { Mean (SD) }\end{array}$} & \multirow{2}{*}{$\begin{array}{l}\geq 8 \mathrm{~h} \text { and/or poor } \\
\text { a } \\
\text { baPWV, } \mathrm{cm} / \mathrm{s} \\
\text { Mean (SD) }\end{array}$} & \multirow[t]{2}{*}{ Adjusted $\beta(95 \% \mathrm{CI})^{\mathrm{b}}$} & \multirow[t]{2}{*}{$P$ for interaction } \\
\hline & & & & & \\
\hline Sex & & & & & 0.089 \\
\hline Male & 5829 & $1693.9(320.6)$ & $1744.9(348.6)$ & $26.8(12.0,41.5)$ & \\
\hline Female & 8656 & $1710.6(338.5)$ & $1764.4(348.8)$ & $6.8(-4.6,18.3)$ & \\
\hline Age, years & & & & & 0.427 \\
\hline$<65$ & 7706 & $1606.4(282.3)$ & $1633.3(292.8)$ & $17.4(6.1,28.7)$ & \\
\hline$\geq 65$ & 6779 & $1832.8(347.2)$ & $1872.9(357.4)$ & $22.1(7.0,37.3)$ & \\
\hline Body mass index, $\mathrm{kg} / \mathrm{m}^{2}$ & & & & & 0.448 \\
\hline$<24$ & 6079 & $1740.5(346.3)$ & $1783.8(361.9)$ & $17.6(2.7,32.4)$ & \\
\hline$\geq 24$ & 8366 & $1678.6(318.6)$ & $1735.3(334.6)$ & $13.0(1.5,24.4)$ & \\
\hline $\mathrm{SBP}, \mathrm{mmHg}$ & & & & & 0.955 \\
\hline$<140$ & 8944 & $1615.5(289.6)$ & $1666.0(308.6)$ & $17.7(6.3,29.0)$ & \\
\hline$\geq 140$ & 5421 & $1852.3(345.0)$ & $1904.3(360.0)$ & $14.4(-2.5,31.3)$ & \\
\hline Current smoking & & & & & 0.049 \\
\hline No & 11402 & $1707.7(336.1)$ & $1758.3(345.7)$ & $9.3(-0.8,19.4)$ & \\
\hline Yes & 3049 & $1689.8(314.3)$ & $1751.9(361.5)$ & $34.2(13.7,54.6)$ & \\
\hline Current alcohol drinking & & & & & 0.130 \\
\hline No & 10760 & $1707.4(333.9)$ & $1760.0(346.7)$ & $10.4(0.2,20.7)$ & \\
\hline Yes & 3205 & $1693.9(325.1)$ & $1751.1(358.1)$ & $28.7(9.4,47.9)$ & \\
\hline Physical activity & & & & & 0.552 \\
\hline Mild & 6416 & $1757.7(347.3)$ & $1820.2(367.1)$ & $16.9(2.3,31.5)$ & \\
\hline Moderate & 6047 & $1675.5(319.1)$ & $1714.0(325.6)$ & $14.2(0.9,27.6)$ & \\
\hline Severe & 2011 & $1631.4(294.4)$ & $1660.3(306.2)$ & $2.9(-18.8,24.7)$ & \\
\hline $\mathrm{TC}(\mathrm{mmol} / \mathrm{L})$ & & & & & 0.098 \\
\hline$<5.2$ & 7117 & $1681.4(321.9)$ & $1739.1(347.4)$ & $23.0(10.4,35.5)$ & \\
\hline$\geq 5.2$ & 7112 & 1727.9 (341.4) & $1774.5(349.1)$ & $6.9(-6.1,19.8)$ & \\
\hline Glucose $(\mathrm{mmol} / \mathrm{L})$ & & & & & 0.167 \\
\hline$<6.1$ & 9174 & $1678.2(320.5)$ & $1733.1(341.8)$ & $18.8(7.8,29.9)$ & \\
\hline$\geq 6.1$ or diabetes ${ }^{c}$ & 5072 & $1753.0(348.2)$ & $1799.2(357.5)$ & $6.9(-8.9,22.6)$ & \\
\hline tHcy $(\mu \mathrm{mol} / \mathrm{L})$ & & & & & 0.400 \\
\hline$<12.2$ & 7159 & $1663.9(315.2)$ & $1714.9(330.4)$ & $10.3(-1.8,22.4)$ & \\
\hline$\geq 12.2$ & 7165 & $1746.1(344.0)$ & $1795.2(360.1)$ & $18.1(4.6,31.6)$ & \\
\hline
\end{tabular}

baPWV brachial-ankle pulse-wave velocity, $C I$ confidence interval

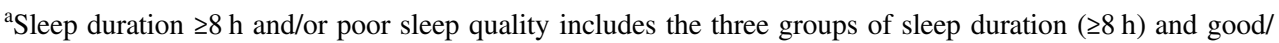
medium sleep quality, sleep duration $(\geq 8 \mathrm{~h})$ and poor sleep quality, and sleep duration $(<8 \mathrm{~h})$ and poor sleep quality

${ }^{b}$ Each subgroup analysis adjusted, if not stratified, for age, sex, body mass index, triglyceride, total cholesterol (TC), high-density lipoprotein cholesterol, fasting glucose, total homocysteine (tHcy), creatinine, smoking status, alcohol consumption, physical activity, systolic blood pressure (SBP), heart rate, treatment group, and study centers

${ }^{\mathrm{c}}$ Diabetes was defined as fasting serum glucose $\geq 7.0 \mathrm{mmol} / \mathrm{L}$ or self-reported use of hypoglycemic agents or insulin, or physician diagnosed diabetes
73 subjects with poor sleep quality were included in the study; therefore, the power to identify the association was poor. Recently, the HSCAA cohort study [8], including 306 patients with cardiovascular risks, found that low sleep quality was associated with the progression of arterial stiffness over a 3-year follow-up period, the OR of which was $(3.62 ; 95 \% \mathrm{CI}: 1.04-12.55)$. However, no specific scientific discussions were available regarding this association among hypertensive patients in these findings. Our results filled the knowledge gap in this field. The exact mechanisms behind the association between sleep quality and arterial stiffness remain inconclusive. Previous studies have suggested that insulin resistance [33], increased sympathetic activity [34] and endothelial dysfunction [35] may be the potential mechanisms underlying this association. Further studies are warranted to identify more potential mechanisms.

Third, we investigated the combined effect of sleep duration and sleep quality on baPWV levels and indicated that longer sleep duration and/or poor sleep quality was associated with increased baPWV levels and arterial stiffness. Moreover, participants with longer sleep 
durations and poor sleep quality had the highest baPWV levels and prevalence risk of arterial stiffness compared with the other groups. The findings suggest that poor sleep quality may increase the risk of arterial stiffness resulting from longer sleep duration. Regarding the combined effect of sleep duration and sleep quality on the risk of arterial stiffness, the combined effect was not statistically significant in the group with longer sleep duration and poor sleep quality, although the combined risk was the highest (OR, 1.33; 95\% CI, 0.94, 1.89) (Supplemental Table 2). This might be due to the small number of participants in the group $(n=217)$. Stratified analysis revealed that the association between combined sleep duration and sleep quality (sleep duration $\geq 8 \mathrm{~h}$ and/or poor sleep quality vs. sleep duration $<8 \mathrm{~h}$ and good/medium sleep quality) with baPWV was stronger among current smokers. Previous studies have reported that both acute and chronic smoking were positively associated with increased arterial stiffness [36], whereas there was a reversible effect of smoking cessation on arterial stiffness [37]. The exact mechanisms underlying the effect of current smoking on this association remain unclear. A plausible biological explanation may be that abnormal sleep conditions and smoking might share several of the same cellular and molecular mechanisms, including insulin resistance and inflammation, for the pathogenesis of arterial stiffness. Further studies are needed to verify this hypothesis.

Several limitations should not be ignored. First, this was a cross-sectional analysis of the CSPPT and could not clarify the causal relationship between sleep conditions and higher baPWV levels. Second, the present study only explored the association of self-reported sleep conditions, but not objective measures of sleep conditions, with baPWV levels. As several previous studies have reported that there were discrepancies between self-reported and objective measures of sleep conditions when assessing the associations between sleep conditions and hypertension and metabolic syndrome [38, 39], further studies concerning the relationship of objective measures of sleep conditions and baPWV levels are needed to confirm our findings. Finally, the association between sleep conditions and baPWV levels was observed only among treated hypertensive patients in our study, so the findings might not be applicable to other populations.

\section{Conclusions}

In summary, a longer sleep duration $(\geq 8 \mathrm{~h})$ or poor sleep quality was associated with increased baPWV levels and arterial stiffness in hypertensive patients. Maintaining proper sleep duration and good sleep quality may play a positive role in maintaining a relatively low level of arterial stiffness among hypertensive patients. If further confirmed, evaluations of sleep duration and quality along with other known risk factors could help to further identify hypertensive patients at high risk of arterial stiffness.

Acknowledgements We gratefully acknowledge the investigators and participants of the China Stroke Primary Prevention Trial (CSPPT), the parent study, who made this research possible.

Funding This work was supported by funding from the following: the National Key Research and Development Program (2016YFE0205400, 2018ZX09739, 2018ZX09301034003); the Projects of the National Natural Science Foundation of China (81730019, 81973133, 81560079, 81860058, 81960074, 81500233); the Major Projects of the Science and Technology Department, Jiangxi (20152ACB20022, 20171BAB205008); Jiangxi Outstanding Person Foundation (20192BCBL23024); the Outstanding Youths Development Scheme of Nanfang Hospital, Southern Medical University (2017J009); the Science and Technology Planning Project of Guangzhou, China (201707020010); the Science, Technology and Innovation Committee of Shenzhen (JSGG20170412155639040, GJHS20170314114526143, JSGG20180703155 802047); and the Economic, Trade and Information Commission of Shenzhen Municipality [20170505161556110, 20170505160926390].

Author contributions $\mathrm{HH}, \mathrm{HL}, \mathrm{XX}$ and $\mathrm{XQ}$ conceived and designed the study; HH, HL, XQ, LL and CL contributed to the statistical analysis; and HH, HL, PL, XQ and XW drafted the manuscript. All authors contributed to collecting data and reviewed/edited the manuscript for important intellectual content. All authors read and approved the final manuscript.

\section{Compliance with ethical standards}

Conflict of interest All authors have completed the ICMJE uniform disclosure form and have declared the following: XX reports grants from the National Key Research and Development Program [2016YFE0205400, 2018ZX09739, 2018ZX09301034003]; the Science and Technology Planning Project of Guangzhou, China [201707020010]; the Science, Technology and Innovation Committee of Shenzhen [JSGG20170412155639040, GJHS20170314114526143, JSGG20180703155802047]; and the Economic, Trade and Information Commission of Shenzhen Municipality [20170505161556110, 20170505160926390]. XQ reports grants from the National Natural Science Foundation of China [81730019, 81973133] and Outstanding Youths Development Scheme of Nanfang Hospital, Southern Medical University [2017J009]. PL reports grants from the National Natural Science Foundation of China [81560079, 81860058], Major Projects of the Science and Technology Department, Jiangxi [20152ACB20022], the Funding Scheme for Academic and Technical Leaders of Major Disciplines, Jiangxi [20172BCB22027], and the Special Funds for Guiding Local Scientific and Technological Development by the Central Government of China (S2019CXSFG0016). XH reports grants from the National Natural Science Foundation of China [81960074, 81500233]; Jiangxi Outstanding Person Foundation [20192BCBL23024]; and Major Projects of the Science and Technology Department, Jiangxi [20171BAB205008]. No other financial or nonfinancial disclosures were reported.

Publisher's note Springer Nature remains neutral with regard to jurisdictional claims in published maps and institutional affiliations. 
Open Access This article is licensed under a Creative Commons Attribution 4.0 International License, which permits use, sharing, adaptation, distribution and reproduction in any medium or format, as long as you give appropriate credit to the original author(s) and the source, provide a link to the Creative Commons license, and indicate if changes were made. The images or other third party material in this article are included in the article's Creative Commons license, unless indicated otherwise in a credit line to the material. If material is not included in the article's Creative Commons license and your intended use is not permitted by statutory regulation or exceeds the permitted use, you will need to obtain permission directly from the copyright holder. To view a copy of this license, visit http://creativecommons. org/licenses/by/4.0/.

\section{References}

1. van Popele NM, Grobbee DE, Bots ML, Asmar R, Topouchian J, Reneman RS, et al. Association between arterial stiffness and atherosclerosis: the Rotterdam Study. Stroke. 2001;32:454-60.

2. Mattace-Raso FU, van der Cammen TJ, Hofman A, van Popele NM, Bos ML, Schalekamp MA, et al. Arterial stiffness and risk of coronary heart disease and stroke: the Rotterdam Study. Circulation. 2006;113:657-63.

3. Carreras A, Zhang SX, Peris E, Qiao Z, Gileles-Hillel A, Li RC, et al. Chronic sleep fragmentation induces endothelial dysfunction and structural vascular changes in mice. Sleep. 2014;37:1817-24.

4. Calvin AD, Covassin N, Kremers WK, Adachi T, Macedo P, Albuquerque FN, et al. Experimental sleep restriction causes endothelial dysfunction in healthy humans. J Am Heart Assoc. 2014;3:e01143.

5. Yoshioka E, Saijo Y, Kita T, Okada E, Satoh H, Kawaharada M, et al. Relation between self-reported sleep duration and arterial stiffness: a cross-sectional study of middle-aged Japanese civil servants. Sleep. 2011;34:1681-6.

6. Tsai TC, Wu JS, Yang YC, Huang YH, Lu FH, Chang CJ. Long sleep duration associated with a higher risk of increased arterial stiffness in males. Sleep. 2014;37:1315-20.

7. Osonoi Y, Mita T, Osonoi T, Saito M, Tamasawa A, Nakayama S, et al. Poor sleep quality is associated with increased arterial stiffness in Japanese patients with type 2 diabetes mellitus. BMC Endocr Disord. 2015;15:29-35.

8. Kadoya M, Kurajoh M, Kakutani-Hatayama M, Morimoto A, Miyoshi A, Kosaka-Hamamoto K, et al. Low sleep quality is associated with progression of arterial stiffness in patients with cardiovascular risk factors: HSCAA study. Atherosclerosis. 2018;270:95-101.

9. Kim CW, Chang Y, Zhao D, Cainzos-Achirica M, Ryu S, Jung HS, et al. Sleep duration, sleep quality, and markers of subclinical arterial disease in healthy men and women. Arterioscler Thromb Vasc Biol. 2015;35:2238-45.

10. Fan F, Yuan Z, Qin X, Li J, Zhang Y, Li Y, et al. Optimal systolic blood pressure levels for primary prevention of stroke in general hypertensive adults: findings from the CSPPT (China Stroke Primary Prevention Trial). Hypertension. 2017;69:697-704.

11. Fan F, Qi L, Jia J, Xu X, Liu Y, Yang Y, et al. Noninvasive central systolic blood pressure is more strongly related to kidney function decline than peripheral systolic blood pressure in a Chinese community-based population. Hypertension. 2016;67:1166-72.

12. Kiełbasa G, Stolarz-Skrzypek K, Pawlik A, Łątka M, Drożdż T, Olszewska M, et al. Assessment of sleep disorders among patients with hypertension and coexisting metabolic syndrome. Adv Med Sci. 2016;61:261-8.

13. Barbaro NR, Fontana V, Modolo R, De Faria AP, Sabbatini AR, Fonseca $\mathrm{FH}$, et al. Increased arterial stiffness in resistant hypertension is associated with inflammatory biomarkers. Blood Press. 2015;24:7-13.

14. Tsuchikura S, Shoji T, Kimoto E, Shinohara K, Hatsuda S, Koyama $\mathrm{H}$, et al. Brachial-ankle pulse wave velocity as an index of central arterial stiffness. J Atheroscler Thromb. 2010;17:658-65.

15. Yamashina A, Tomiyama H, Takeda K, Tsuda H, Arai T, Hirose $\mathrm{K}$, et al. Validity, reproducibility, and clinical significance of noninvasive brachial-ankle pulse wave velocity measurement. Hypertens Res. 2002;25:359-64.

16. Huo Y, Li J, Qin X, Huang Y, Wang X, Gottesman RF, et al. CSPPT Investigators. Efficacy of folic acid therapy in primary prevention of stroke among adults with hypertension in China: the CSPPT randomized clinical trial. JAMA. 2015;313:1325-35.

17. Qin X, Li Y, He M, Tang G, Yin D, Liang M, et al. Folic acid therapy reduces serum uric acid in hypertensive patients: a substudy of the China Stroke Primary Prevention Trial (CSPPT). Am J Clin Nutr. 2017;105:882-9.

18. Qin X, Li J, Zhang Y, Chen D, Wang B, He M, et al. Effect of folic acid supplementation on risk of new-onset diabetes in adults with hypertension in China: Findings from the China Stroke Primary Prevention Trial (CSPPT). J Diabetes. 2016;8:286-94.

19. Qin X, Shen L, Zhang R, Li Y, Wang X, Wang B, et al. Effect of folic acid supplementation on cancer risk among adults with hypertension in China: a randomized clinical trial. Int J Cancer. 2017;141:837-47.

20. Zheng M, Huo Y, Wang X, Xu X, Qin X, Tang G, et al. A prospective study on pulse wave velocity (PWV) and response to anti-hypertensive treatments: PWV determines BP control. Int $\mathbf{J}$ Cardiol. 2015;178:226-31.

21. Song $\mathrm{Y}, \mathrm{Xu} \mathrm{B}, \mathrm{Xu} \mathrm{R}$, Tung R, Frank E, Tromble W, et al. Independent and joint effect of brachial-ankle pulse wave velocity and blood pressure control on incident stroke in hypertensive adults. Hypertension. 2016;68:46-53.

22. Kitagawa N, Ushigome E, Matsumoto S, Oyabu C, Ushigome H, Yokota I, et al. Threshold value of home pulse pressure predicting arterial stiffness in patients with type 2 diabetes: KAMOGAWAHBP study. J Clin Hypertens. 2018;20:472-7.

23. Takashima N, Turin TC, Matsui K, Rumana N, Nakamura Y, Kadota A, et al. The relationship of brachial-ankle pulse wave velocity to future cardiovascular disease events in the general Japanese population: the Takashima Study. J Hum Hypertens. 2014;28:323-7.

24. He M, Qin X, Cui Y, Cai Y, Sun L, Xu X, et al. Prevalence of unrecognized lower extremity peripheral arterial disease and the associated factors in Chinese hypertensive adults. Am J Cardiol. 2012;110:1692-8.

25. Qin X, Li J, Zhang Y, Ma W, Fan F, Wang B, et al. Prevalence and associated factors of diabetes and impaired fasting glucose in Chinese hypertensive adults aged 45 to 75 years. PLoS ONE. 2012;7:e42538.

26. Qin X, Zhang Y, Cai Y, He M, Sun L, Fu J, et al. Prevalence of obesity, abdominal obesity and associated factors in hypertensive adults aged 45-75 years. Clin Nutr. 2013;32:361-7.

27. Cao X, Zhou J, Yuan H, Chen Z. Association between sleep condition and arterial stiffness in Chinese adult with nonalcoholic fatty liver disease. J Thromb Thrombolysis. 2016;42:127-34.

28. Jike M, Itani O, Watanabe N, Buysse DJ, Kaneita Y. Long sleep duration and health outcomes: a systematic review, meta-analysis and meta-regression. Sleep Med Rev. 2018;39:25-36.

29. Grandner MA, Drummond SP. Who are the long sleepers? Towards an understanding of the mortality relationship. Sleep Med Rev. 2007;11:341-60.

30. Irwin MR, Olmstead R, Carroll JE. Sleep disturbance, sleep duration, and inflammation: A systematic review and metaanalysis of cohort studies and experimental sleep deprivation. Biol Psychiatry. 2016;80:40-52. 
31. Mozos I, Malainer C, Horbańczuk J, Gug C, Stoian D, Luca CT, et al. Inflammatory markers for arterial stiffness in cardiovascular disease. Front Immunol. 2017;8:1058.

32. Guney I, Akgul YS, Gencer V, Aydemir H, Aslan U, Ecirli S. Sleep quality and risk factors of atherosclerosis in predialysis chronic kidney disease. J Artif Organs. 2017;39:563-9.

33. Kline CE, Hall MH, Buysse DJ, Earnest CP, Church TS. Poor sleep quality is associated with insulin resistance in postmenopausal women with and without metabolic syndrome. Metab Syndr Relat Disord. 2018;16:183-9.

34. Zhang J, Ma RC, Kong AP, So WY, Li AM, Lam SP, et al. Relationship of sleep quantity and quality with 24-hour urinary catecholamines and salivary awakening cortisol in healthy middleaged adults. Sleep. 2011;34:225-33.

35. Cooper DC, Ziegler MG, Milic MS, Ancoli-Israel S, Mills PJ, Loredo JS, et al. Endothelial function and sleep: associations of flow-mediated dilation with perceived sleep quality and rapid eye movement (REM) sleep. J Sleep Res. 2014;23:84-93.

36. Doonan RJ, Hausvater A, Scallan C, Mikhailidis DP, Pilote L, Daska-lopoulou SS. The effect of smoking on arterial stiffness. Hypertens Res. 2010;33:398-410.

37. Takami T, Saito Y. Effects of smoking cessation on central blood pressure and arterial stiffness. Vasc Health Risk Manag. 2011;7: 633-8.

38. Ren R, Covassin N, Yang L, Li Y, Zhang Y, Zhou J, et al. Objective but not subjective short sleep duration is associated with hypertension in obstructive sleep apnea. Hypertension. 2018;72: 610-7.

39. Bowman MA, Duggan KA, Brindle RC, Kline CE, Krafty RT, Thayer JF, et al. Prospective associations among objectively and subjectively assessed sleep and the metabolic syndrome. Sleep Med. 2019;58:1-6. 